\title{
NEURO-CPT: CLASSIFICAÇÃO DE SOLOS USANDO-SE REDES NEURAIS ARTIFICIAIS
}

\author{
Luiz Biondi Neto ${ }^{1}$ \\ Ana Cristina Castro F. Sieira ${ }^{1}$ \\ Bernadete Ragoni Danziger ${ }^{1}$ \\ José Guilherme Santos da Silva ${ }^{1}$
}

\begin{abstract}
Resumo: Este trabalho é uma aplicação da inteligência computacional na área de geotecnia, especificamente na classificação de solos. Os dados usados no treinamento da Rede Neural Artificial (RNA) foram obtidos de situações reais, com a classificação validada pelo método de determinação do comportamento do solo através do Teste da Penetração do Cone (CPT). Não foram usados nessa investigação, dados referentes à pressão intersticial da água (poropressão) empregados na correção do valor da resistência da ponta do cone no método piezocone com medição da poropressão $(\mathrm{CPTu})$. Assim a variável de entrada $\mathrm{q}_{\mathrm{c}}$, que indica a resistência da ponta do cone e Rf, razão de atrito, que representa a relação entre a resistência de atrito lateral $f_{\mathrm{s}}$ e a resistência da ponta do cone, são usadas para mapear 12 tipos de solos, abrangendo desde solos finos e sensíveis a areias pedregulhosas. O banco de dados utilizado para teste também foi devidamente validado pelo teste CPT e os resultados obtidos pela RNA, treinada pelo método resiliente de retro propagação de erro apresentaram uma percentagem de acerto de classificação do solo, próxima de $98 \%$.
\end{abstract}

Palavras-chave:Classificação de solo; Teste de penetração do cone; RNA; Método Resiliente.

\begin{abstract}
This work is a computational intelligence application in the geotechnical area, specifically in soils classification. The training data used in the Artificial Neural Networks (ANN) was obtained based on real situations, with the classification validated by method of soil characterization through the Cone Penetration Test (CPT). In this contribution pore pressures data from CPTu (CPT test with pore pressure measurement) was not included. The pore pressures data (interstitial water pressure) is commonly used to correct $\mathrm{q}_{\mathrm{c}}$ (cone tip resistance) in CPTu tests. Therefore, the input variables obtained from the cone penetration test and employed in the present investigation are only the $\mathrm{q}_{\mathrm{c}}$, the cone tip resistance, and $\mathrm{Rf}$, the friction ratio. These two variables were used to map 12 different soils types, ranging from sensible fine grained soils to gravel sands. The test database was properly validated by the CPT and the results obtained by (ANN), using back propagation resilient method, revealed a successful capability in soil classification, presenting a percentage of $98 \%$ of agreement with the CPT method.
\end{abstract}

Keywords: Soil behavior; Cone Penetration Test; ANN; Resilient propagation method.

\footnotetext{
${ }^{1}$ Universidade do Estado do Rio de Janeiro - UERJ, Programa de Pós-Graduação em Engenharia Civil PGECIV-luizbiondi@terra.com.br; anacriscastro@hotmail.com; brdanzig@uerj.br; jgss@uerj.br. 


\section{INTRODUÇÃO}

A elaboração de projetos geotécnicos exige um conhecimento adequado das características e das propriedades geotécnicas dos solos. $\mathrm{O}$ reconhecimento do subsolo e a determinação das propriedades geotécnicas podem ser feitos através de investigações geotécnicas de campo. Na maioria dos casos, são necessárias a utilização de correlações empíricas e calibrações para converter os resultados de ensaios de campo em propriedades adequadas para o projeto. Dentre os principais métodos de investigação geotécnica, destaca-se o ensaio de penetração do cone - CPT. Os resultados destes ensaios permitem a identificação estratigráfica de perfis de solos, a determinação de suas propriedades mecânicas, estimadas com base em correlações empíricas e semi-empíricas, visando aplicações em diversas áreas da geotecnia, em particular na previsão da capacidade de carga e de recalques de fundações BUDHU (2006), BARNES (2000) e CHEN (2002).

Os primeiros estudos relativos ao ensaio de penetração estática, chamado de "diepsondering", "deepsounding", ensaio de cone holandês ou simplesmente ensaio de cone, foram realizados por TERZAGHI (1930), segundo VELLOSO (1959). Logo em seguida, estudos se seguiram através dos especialistas do Laboratório de Mecânica dos Solos de Delft, na Holanda, VELLOSO (1959). Na década de 30, foi desenvolvido um medidor mecânico de penetração de cone, que posteriormente sofreu modificações importantes introduzidas por BEGEMANN (1953, 1963), um dos pioneiros no estudo do ensaio de penetração estática.

Em seus estudos, BEGEMANN (1965) mostrou ser possível classificar o solo em função da razão entre a resistência de atrito lateral $\left(f_{s}\right)$ e a resistência de ponta $\left(\mathrm{q}_{\mathrm{c}}\right)$ do cone, definida pelo parâmetro $\mathrm{Rf}$ (razão de atrito). A Tabela 1 apresenta a classificação dos solos proposta por BEGEMANN (1965), em função do parâmetro Rf.

Tabela 1-Classificação do solo em função da razão de atrito, segundo BEGEMANN (1965)

\begin{tabular}{|l|c|}
\hline $\begin{array}{l}\text { CLASSIFICAÇÃO } \\
\text { DO SOLO }\end{array}$ & $\begin{array}{c}\text { RAZÃO DE } \\
\text { ATRITO (\%) }\end{array}$ \\
\hline $\begin{array}{l}\text { Areia Grossa com } \\
\text { Cascalhos à Areia Fina }\end{array}$ & 1,2 a 1,6 \\
\hline Areia Siltosa & 1,6 a 2,2 \\
\hline Areia Silto-Argilosa & 2,2 a 3,2 \\
\hline Misturas de Argila & 3,2 a 4,1 \\
\hline Argila & 4,1 a 7,0 \\
\hline Turfa & $>7,0$ \\
\hline
\end{tabular}

Algumas modificações realizadas posteriormente conduziram à introdução de sensores, objetivando a medição dos parâmetros de interesse, sendo este dispositivo denominado de medidor elétrico de penetração do cone. Outros autores como SANGLERAT (1974) e SEARLE (1979), tomando por base o cone mecânico, ampliaram e modificaram a proposta original de BEGEMANN (1953, 1963 e 1965). As mudanças introduzidas foram no sentido de controlar a velocidade de penetração do cone, variar as dimensões da haste e do cone e aumentar a profundidade das sondagens. Algumas variáveis adicionais também foram posteriormente introduzidas, como a medida da pressão intersticial da água, poropressão, dando origem ao piezocone $(\mathrm{CPTu})$, sucessor do cone holandês. Os principais trabalhos que relatam essa evolução podem ser encontrados em SANGLERAT (1974) e DANZIGER (1990).

No Brasil, este tipo de ensaio, regido pela ABNT (1991), é aplicado desde a década 1950 , como ensaio de penetração 
estática mecânico, ganhando impulso a partir de 1980, com a intensificação de pesquisas relacionadas ao tema, ROCHA (1982), DANZIGER (1986) e DANZIGER (1998).

Uma das aplicações do cone consiste na identificação do perfil geotécnico a partir do emprego de ábacos de classificação. As várias propostas de classificação dos solos, a partir dos resultados dos ensaios de cone, baseiam-se, em geral, em ábacos que relacionam a resistência de ponta $\mathrm{q}_{\mathrm{c}} \mathrm{e}$ a razão de atrito Rf, ESLAMI (1995, 1996 e 1997)

Atualmente, a determinação do tipo de solo através de ábacos vem sendo substituída por programas computacionais, envolvendo métodos estatísticos de classificação. No entanto, as classes de solos são linearmente não separáveis podendo, em certas situações, dificultar o trabalho estatístico de classificação. Além disso, por se tratar de um processo de aquisição e transmissão de dados emitidos de sensores para um sistema elétrico, podem ocorrer perdas desses dados no ensaio, sendo muito difícil a recomposição e a classificação correta do solo na profundidade para qual ocorreu a falha.

Neste sentido, as Redes Neurais Artificiais (RNAs), que são estruturas paralelas compostas de neurônios artificiais, distribuídos em camadas, densamente conectados, podem ser muito úteis. As RNAs apresentam duas fases distintas de operação: a aprendizagem ou treinamento, e a execução. $\mathrm{Na}$ fase de treinamento ou aprendizagem são apresentados estímulos à entrada, denominados padrões de treinamento representados, por exemplo, pelas características $\mathrm{q}_{\mathrm{c}}$ e $\mathrm{Rf}$ dos ensaios e pelos alvos, no caso investigado, os 12 tipos de solos definidos nas classificações pelo método CPT. Na fase de execução, as RNAs, devidamente treinadas, recebem como estímulo de entrada um conjunto de padrões de testes, também representados pelos padrões $\mathrm{q}_{\mathrm{c}}$ e $\mathrm{Rf}$, e que não fizeram parte do treinamento DAYHOFF (1990) e HAYKIN (1994). Essa capacidade é chamada de generalização e pode ser explorada no sentido de obtermos a classificação correta do solo, mesmo que parte dos dados sejam perdidos ZURADA (1992), por qualquer tipo de falha de transmissão.

Finalmente, a RNA apresenta como resultado a classificação do solo para cada incremento de profundidade.

\section{2- FUNDAMENTAÇÃO TEÓRICA}

\section{1 - O ensaio CPT}

$\mathrm{O}$ equipamento consiste num conjunto de hastes tendo na extremidade inferior um cone com ângulo de vértice de $60^{\circ}$ e uma base de $10 \mathrm{~cm}^{2}$ (Figura 1). A resistência de ponta é medida através de uma célula de carga. Um conjunto de hastes metálicas transmite a força de cravação da máquina ao cone WOODWARD (2004), SCHOFIELD (2005), DAVID (2004) e HEAD (2006).

Interiormente às hastes passa o cabo de alimentação do cone, RUITER (1991). A resistência de ponta é obtida continuamente e os valores correspondentes podem ser registrados em gráficos, simultaneamente à realização do ensaio. Aquele autor descreve também a medição da resistência de atrito lateral, através de uma célula de carga fixada à luva de atrito, situada logo acima da base.

O ensaio CPT é normalizado no Brasil ABNT (1991), sendo utilizado de forma crescente em inúmeros projetos geotécnicos. Segundo CAMPANELLA (1982, 1988 e 1989), apenas no início dos anos 80 os elementos de poro-pressão foram incorporados aos cones elétricos, resultando no equipamento designado como piezocone, WALTHAM (2001), VOS (1982) e PRAKASH (1990). 

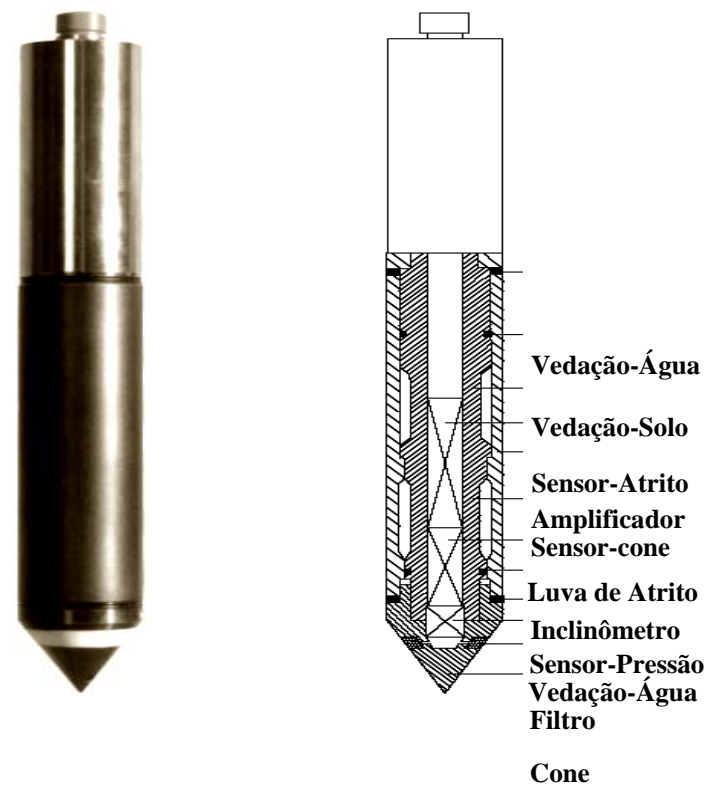

Figura 1 - Cone de penetração para ensaios CPT, adaptado de FUGRO (2000)

O cone é cravado no solo a uma velocidade constante, por um sistema hidráulico instalado em perfuratriz ou em veículos preparados para esta finalidade KAMESWARA (2002), GREGG (1998), SCHMERTMANN (1978) e HSAI-YANG (2006). O equipamento de cravação é uma estrutura de reação na qual é montado o sistema de aplicação de cargas, realizado por mecanismos hidráulicos SOARES (1986). A penetração das hastes de $1 \mathrm{~m}$ de comprimento é feita continuamente e seguida da retração do pistão hidráulico para colocação de nova haste QUARESMA (1996) e DANZIGER (1990).

O conjunto pode ser montado sobre um reboque ou caminhão previamente preparado para esta finalidade, de capacidade entre 100 e $200 \mathrm{kN}$. A reação aos esforços de cravação é obtida pelo peso próprio do equipamento e pela ancoragem manual DANZIGER (1990), DAVIES (1995) e DOUGLAS (1981).
Durante a penetração, as forças medidas pela ponta e pelo atrito lateral variam em função das propriedades dos materiais atravessados. Os registros de $\mathrm{q}_{\mathrm{c}} \mathrm{e}$ $\mathrm{f}_{\mathrm{s}}$ são monitorados continuamente $\mathrm{e}$ digitalizados em intervalos típicos de 10 , 25 e $50 \mathrm{~mm}$. Com base nas medidas de resistência de ponta $\left(\mathrm{q}_{\mathrm{c}}\right)$ e atrito lateral $\left(\mathrm{f}_{\mathrm{s}}\right)$, calcula-se a razão de atrito $\mathrm{Rf}=\mathrm{f}_{\mathrm{s}} / \mathrm{q}_{\mathrm{c}}$. $\mathrm{O}$ conhecimento da resistência à penetração de ponta do cone $q_{c}$ e da relação de atrito Rf permite a determinação do tipo de solo, através do ábaco proposto por ROBERTSON (1983 e 1986). A Figura 2 apresenta o ábaco, que permite a identificação de 12 diferentes tipos de solo.

\begin{tabular}{|c|}
\hline 1 \\
\hline 2 \\
\hline 3 \\
\hline 4 \\
\hline 5 \\
\hline 6 \\
\hline 7 \\
\hline 8 \\
\hline 9 \\
\hline 10 \\
\hline 11 \\
\hline 12 \\
\hline
\end{tabular}

Solo fino sensível Matéria orgânica Argila

Argila siltosa a argila Silte argiloso a argila siltosa Silte arenoso a silte argiloso Areia siltosa a silte arenoso Areia a areia siltosa Areia Areia com pedregulho a areia Solo fino muito rijo Areia a areia argilosa

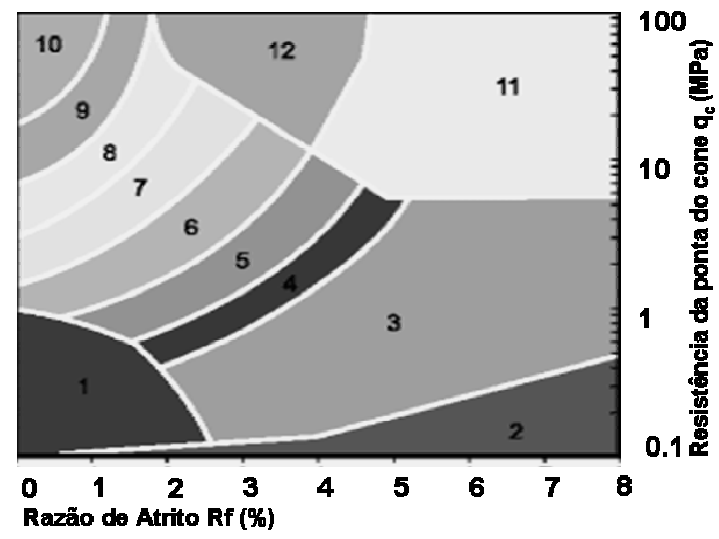

Figura 2 - Classificação do Solo proposta por ROBERTSON (1986 e 1990)

\section{2 - As RNAs e o método Resiliente}

O algoritmo de treinamento mais utilizado no perceptron de múltiplas camadas (MLP), é o de retro propagação de erro (BP), baseado no método do gradiente 
descendente, que computa as derivadas parciais de uma função de erro, com relação ao vetor peso $\mathrm{W}$ de certo vetor de entrada DAYHOFF (1990) e HAYKIN (1994).

A regra de Widrow-Hoff é a base fundamental de diversos métodos de treinamento das RNAs, inclusive MLPs ZURADA (1992). Esta regra avalia o erro (e) a cada iteração através da derivada parcial desse erro em relação ao peso $\mathrm{W}$ e ao limiar (b), mostrados, de forma simples no perceptron da Figura 3.

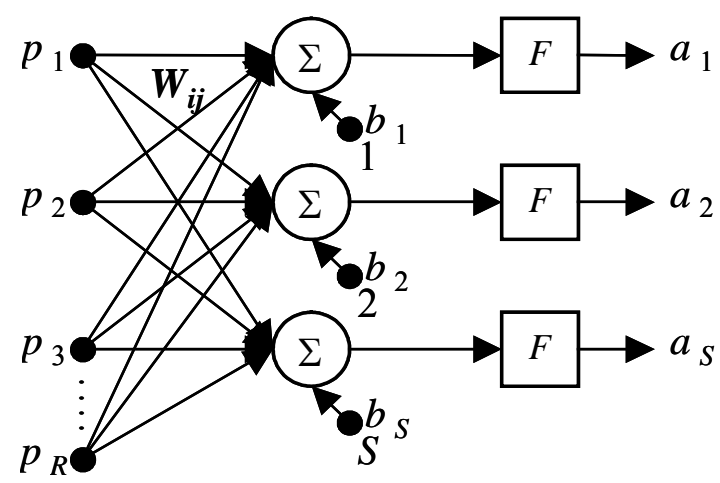

Figura 3 - Rede Neural Artificial

Sabendo-se que (T) representa o vetor alvo a ser atingido no treinamento, (S) o número de elementos do vetor de saída $\left(\mathrm{A}=\left[\begin{array}{llll}a_{2} & a_{2} & \ldots & a_{\mathrm{S}}\end{array}\right]^{\mathrm{t}}\right), \mathrm{R}$ o número de elementos do vetor $\left(\mathrm{P}=\left[\begin{array}{llll}p_{2} & p_{2} & \ldots & p_{\mathrm{R}}\end{array}\right]^{\mathrm{t}}\right)$, apresentado à entrada da rede e $(\mathrm{F})$ a função de ativação e, finalmente, analisando-se um neurônio genérico, para n-ésima iteração, $(j=1, \ldots, R)$, tem-se (1):

$$
\begin{aligned}
& \frac{\partial e^{(n)}}{\partial W_{i j}}=\frac{\partial\left[\mathrm{T}^{(n)}-\mathrm{A}^{(n)}\right]}{\partial W_{i j}}=\frac{\partial}{\partial W_{i j}}\left[\mathrm{~T}^{(n)}-\sum_{j=1}^{R} W_{i j} \mathrm{P}_{j}^{(n)}\right] \\
& \frac{\partial e^{(n)}}{\partial W_{i j}}=-\mathrm{P}_{j}^{(n)}
\end{aligned}
$$

$\mathrm{Na}$ expressão (2) é mostrada a variação $\Delta \mathrm{W}$ do peso e $\mathrm{o}$ processo adaptativo de atualização do parâmetro interno, peso da rede ZURADA (1992).

Análise semelhante poderia ser feita em relação ao limiar (b), outro parâmetro interno da RNA.

$$
\begin{aligned}
& \Delta W_{i j}^{(n)}=-\eta \frac{\partial e^{(n)}}{\partial W_{i j}} \\
& W_{i j}^{(n+1)}=W_{i j}^{(n)}+\Delta W_{i j}^{(n)}=W_{i j}^{(n)}+\eta \mathrm{P}_{j}^{(n)}
\end{aligned}
$$

A técnica de retro propagação de erro, baseada no método do gradiente decrescente, tratado em sua essência nas equações (1) e (2) é normalmente usada no treinamento de redes de múltiplas camadas (MLP) HAYKIN (1994).

Dependendo do mapeamento desejado, o método do gradiente decrescente, que depende da magnitude do gradiente, isto é da magnitude das derivadas parciais do erro (e) sobre o peso pode se tornar lento, especialmente no final do treinamento, inviabilizando aplicações que exigem soluções rápidas DAYHOFF (1990) e HAYKIN (1994).

Por outro lado, o método Resiliente de retro propagação de erro, usado nessa investigação, faz parte de uma classe de estratégias rápidas de adaptação local para treinamento de RNAs e seu algoritmo foi desenvolvido por Riedmiller e Braun, RIEDMILLER (1993).

É um método independente da magnitude do gradiente do erro sobre o peso, no qual a atualização dos pesos depende, simplesmente, do sinal dos termos do gradiente e o aprendizado é feito por épocas. O ajuste dos pesos é realizado depois da apresentação completa de todo o padrão de treinamento à RNA.

No método Resiliente, a variação dos pesos é feita de forma separada. Assim, os pesos $\mathrm{W}_{\mathrm{ij}}$ possuem uma taxa de variação própria $\Delta_{\mathrm{i} j}$, com o tempo, obedecendo a seguinte regra, como mostrado em (3): 
$\Delta_{i j}{ }^{(t)}=\left\{\begin{array}{l}\eta^{+} \Delta_{i j}{ }^{(t-1)} \\ \eta^{-} \Delta_{i j}{ }^{(t-1)} \\ \Delta_{i j}{ }^{(t-1)}\end{array}\right.$

onde : $0<\eta^{-}<1<\eta^{+}$

Os valores típicos para $\eta$ e $\Delta_{\mathrm{ij}}$ são: $\eta^{+}=1.2$ , $\eta^{-}=0.5$ e $10^{-6}<\Delta_{\mathrm{ij}}<50$.

O fato do gradiente do erro sobre o peso não mudar de sinal em duas iterações sucessivas indica que o sistema está se movendo na mesma direção, sugerindo um aumento de $\Delta_{\mathrm{ij}}$ proporcional a $\eta^{+}$, visando acelerar a convergência.

A mudança do sinal do gradiente em duas iterações sucessivas indica que a última atualização do peso foi excessiva, levando o sistema a saltar sobre um mínimo e sugerindo uma diminuição de $\Delta_{\mathrm{ij}}$ proporcional a $\eta^{-}$.

Se o gradiente $\frac{\partial \mathrm{e}^{(t)}}{\partial W_{i j}}$ for positivo, o erro cresce e a variação do peso deve ser decrementada na atualização $\Delta W_{i j}^{(t)}=$ $-\Delta_{i j}{ }^{(t)}$ com conseqüente diminuição do peso.

Se o gradiente $\frac{\partial \mathrm{e}^{(t)}}{\partial W_{i j}}$ é negativo, o erro decresce e a variação do peso deve ser incrementada na atualização $\Delta W_{i j}^{\left({ }^{(t)}\right.}=\Delta_{i j}{ }^{\left({ }^{t}\right)}$ e conseqüentemente aumentando o peso.

Se o gradiente $\frac{\partial \mathrm{e}^{(t)}}{\partial W_{i j}}=0$ não ocorre variação na atualização do peso, isto é, $\Delta W_{i j}^{(t)}=0$.

ENGEVISTA, v. 8, n. 1, p. 37-48, junho 2006
Finalmente, os pesos são atualizados através de (4):

$$
W_{i j}^{(t+1)}=W_{i j}^{(t)}+\Delta W_{i j}^{(t)}
$$

\section{3- MODELAGEM E TREINAMENTO DAS RNAS}

O modelo da RNA que apresentou o melhor desempenho foi a arquitetura composta de duas camadas escondidas. A primeira camada com 10 neurônios, a segunda com 150 neurônios e a saída com 12 neurônios artificiais. A Figura 4 apresenta um diagrama em bloco da RNA, no qual são ressaltadas as entradas e as saídas da RNA.

A rede foi treinada com um banco de dados validado pelo método CPT envolvendo uma matriz de 2 linhas $\left(\mathrm{q}_{\mathrm{c}}\right.$ e Rf) e 545 colunas de exemplos representativos das 12 classes distintas de solos a serem tipificados.

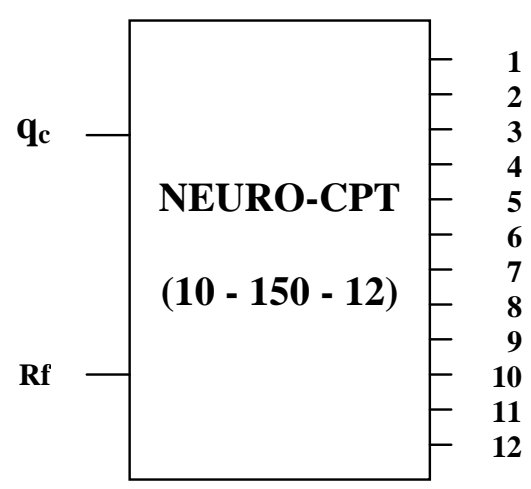

Figura 4 - Diagrama do modelo da RNA

Os parâmetros de treinamento da RNA foram os seguintes:

Número máximo de épocas $=1000$

Tolerância do erro $=1 \mathrm{e}-3$

Os parâmetros $\eta^{+}, \eta^{-}$e $\Delta_{i j}$ têm seus valores especificados pelo método resiliente, tratado na seção 2.2 .

A Figura 5 mostra a curva de treinamento da rede na qual pode ser visto 
que o processo convergiu muito rapidamente ao atingir cerca de 230 épocas.

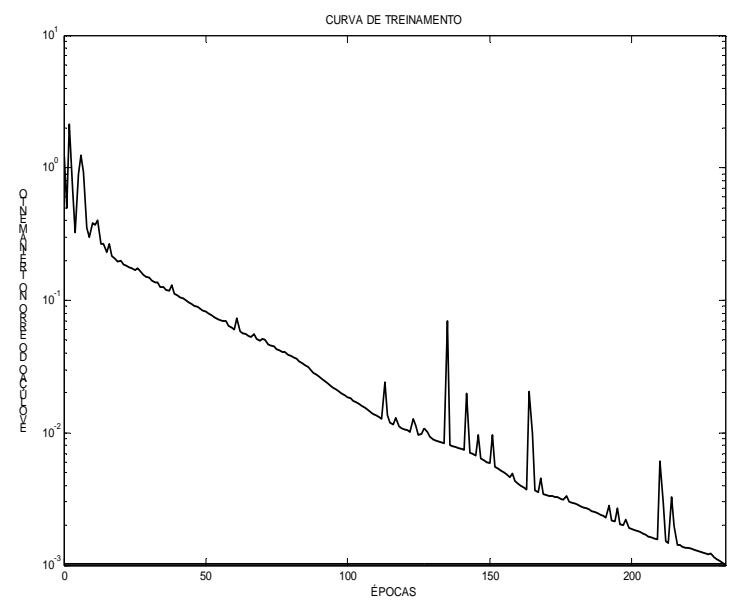

Figura 5 - Curva de Treinamento

\section{4- RESULTADOS}

Depois de devidamente treinada, a RNA foi submetida a diversos testes, visando verificar a coerência dos seus resultados. A validação dos resultados obtidos pela rede é verificada pela comparação com a classificação obtida pela aplicação direta do método de interpretação do CPT, que usa o ábaco mostrado na Figura 2.

A Figura 6 apresenta 462 padrões de testes $\left(\mathrm{q}_{\mathrm{c}}\right.$ e $\left.\mathrm{Rf}\right)$ do ensaio CPT usado no teste. $\mathrm{O}$ incremento da penetração do cone utilizado no ensaio foi de $0.05 \mathrm{~m}$, até uma profundidade máxima de $23 \mathrm{~m}$ de penetração, com uma velocidade de penetração controlada de cerca de 20 $\mathrm{mm} / \mathrm{s}$.

Como a função de ativação na camada de saída da RNA é a tangente hiperbólica, tendo seus limites entre -1 e 1 , a classificação é considerada válida se a saída da rede correspondente ao solo classificado atingir valor maior ou igual a + 0.85 e todas as demais atingirem valores menores ou iguais a -0.85 , Tabela 2. Caso contrário o solo não será classificado corretamente.

Tabela 2 - Saída da RNA para 5 dos 462 pares de teste da sondagem

\begin{tabular}{|l|c|c|c|c|c|}
\hline \# 1 & $\mathbf{- 1 . 0 0 0}$ & $\mathbf{- 1 . 0 0 0}$ & $\mathbf{- 1 . 0 0 0}$ & $\mathbf{- 1 . 0 0 0}$ & $\mathbf{- 1 . 0 0 0}$ \\
\hline \# 2 & -1.000 & -1.000 & -1.000 & -1.000 & -1.000 \\
\hline \# 3 & -1.000 & -1.000 & -1.000 & -0.999 & -1.000 \\
\hline \# 4 & -0.997 & -1.000 & -0.987 & $\mathbf{0 . 9 8 3}$ & -0.997 \\
\hline \# 5 & $\mathbf{0 . 9 9 4}$ & -0.999 & $\mathbf{0 . 9 9 4}$ & -0.987 & $\mathbf{0 . 9 7 3}$ \\
\hline \# 6 & -0.999 & $\mathbf{0 . 9 9 9}$ & -1.000 & -1.000 & -0.991 \\
\hline \# 7 & -0.999 & -0.989 & -1.000 & -1.000 & -0.999 \\
\hline \# 8 & -1.000 & -1.000 & -1.000 & -1.000 & -1.000 \\
\hline \# 9 & -1.000 & -1.000 & -1.000 & -1.000 & -1.000 \\
\hline \# 10 & -1.000 & -1.000 & -1.000 & -1.000 & -1.000 \\
\hline \# 11 & -1.000 & -1.000 & -1.000 & -1.000 & -1.000 \\
\hline \# 12 & -1.000 & -0.983 & -1.000 & -1.000 & -1.000 \\
\hline TIPO & $\mathbf{5}$ & $\mathbf{6}$ & $\mathbf{5}$ & $\mathbf{4}$ & $\mathbf{5}$ \\
\hline
\end{tabular}



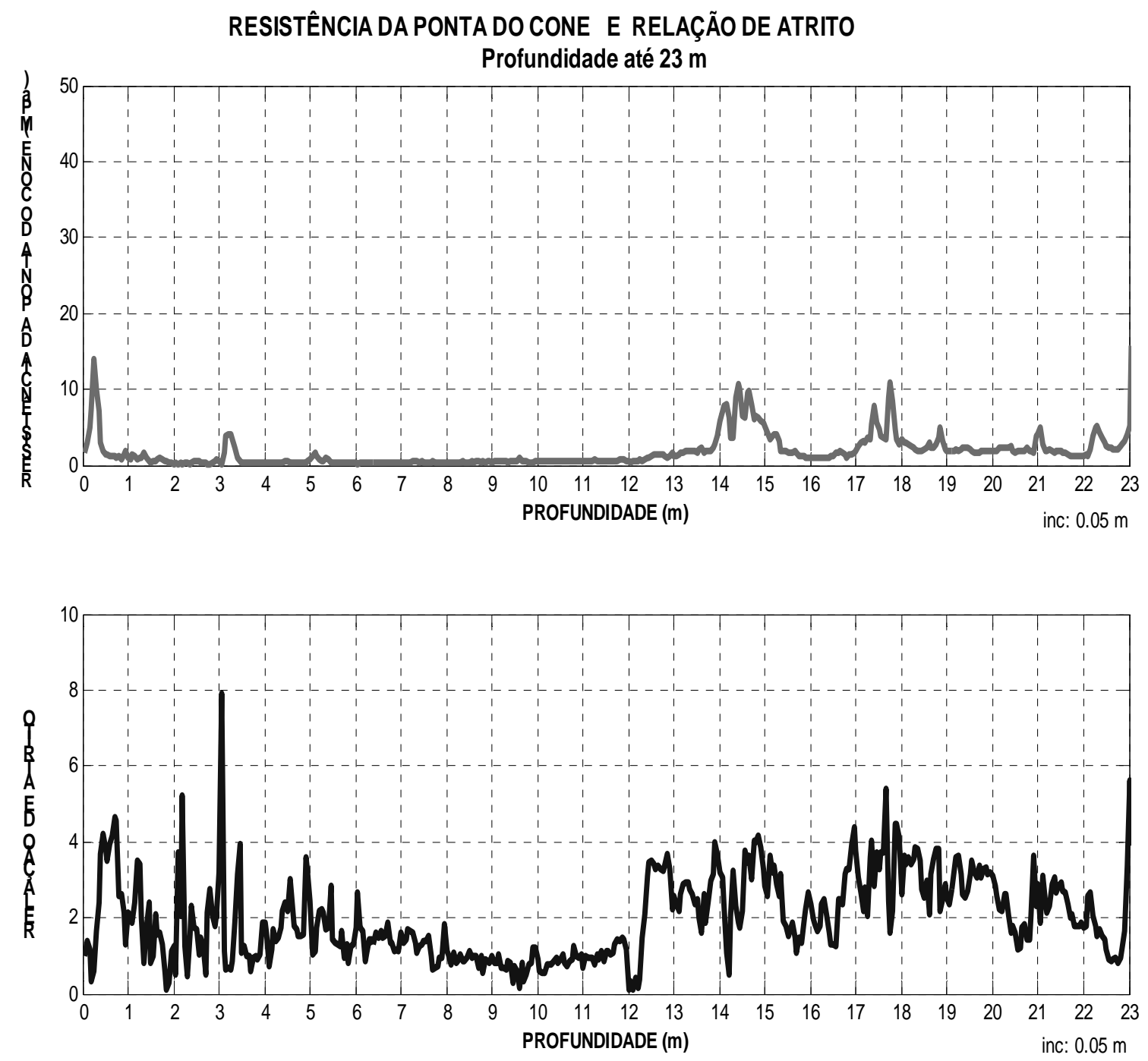

Figura 6 - Padrões de teste

Finalmente, a Figura 7 apresenta o resultado completo da interpretação do ensaio obtido através da RNA. Nesta figura pode ser vista a classificação (tipo 1 a 12), em função da profundidade (até $23 \mathrm{~m}$ ).

\section{5- CONCLUSÕES}

O presente trabalho apresentou uma aplicação da inteligência computacional na área de Geotecnia, especificamente no que se refere à classificação dos solos a partir de resultados de ensaios de penetração do cone.

ENGEVISTA, v. 8, n. 1, p. 37-48, junho 2006
A comparação entre os resultados da classificação do solo obtidos através da RNA, submetida aos padrões de teste, e o método empírico proposto por CAMPANELLA $(1982,1988$ e 1989) para a interpretação dos resultados de ensaios de cone (CPT), apresentou um erro percentual inferior a $2 \%$.

O método resiliente de propagação se mostrou bastante adequado para $\mathrm{o}$ treinamento da RNA. Comparando o tempo de convergência com o método do gradiente tradicional, o método resiliente foi cerca de 10 vezes mais rápido, facilitando os ajustes dos parâmetros da rede, permitindo a realização de muitos testes com diferentes configurações. 
Ficou evidente através do teste de generalização, que o método resiliente além de rápido, é bastante confiável, pois o erro de classificação se mostrou muito pequeno quando comparado com o método de retro propagação de erro tradicional.

No momento, os autores desta investigação estão trabalhando no sentido de treinar uma nova RNA, baseada agora em padrões obtidos pelo método piezocone com medição da pressão intersticial (poropressão) da água (CPTu).

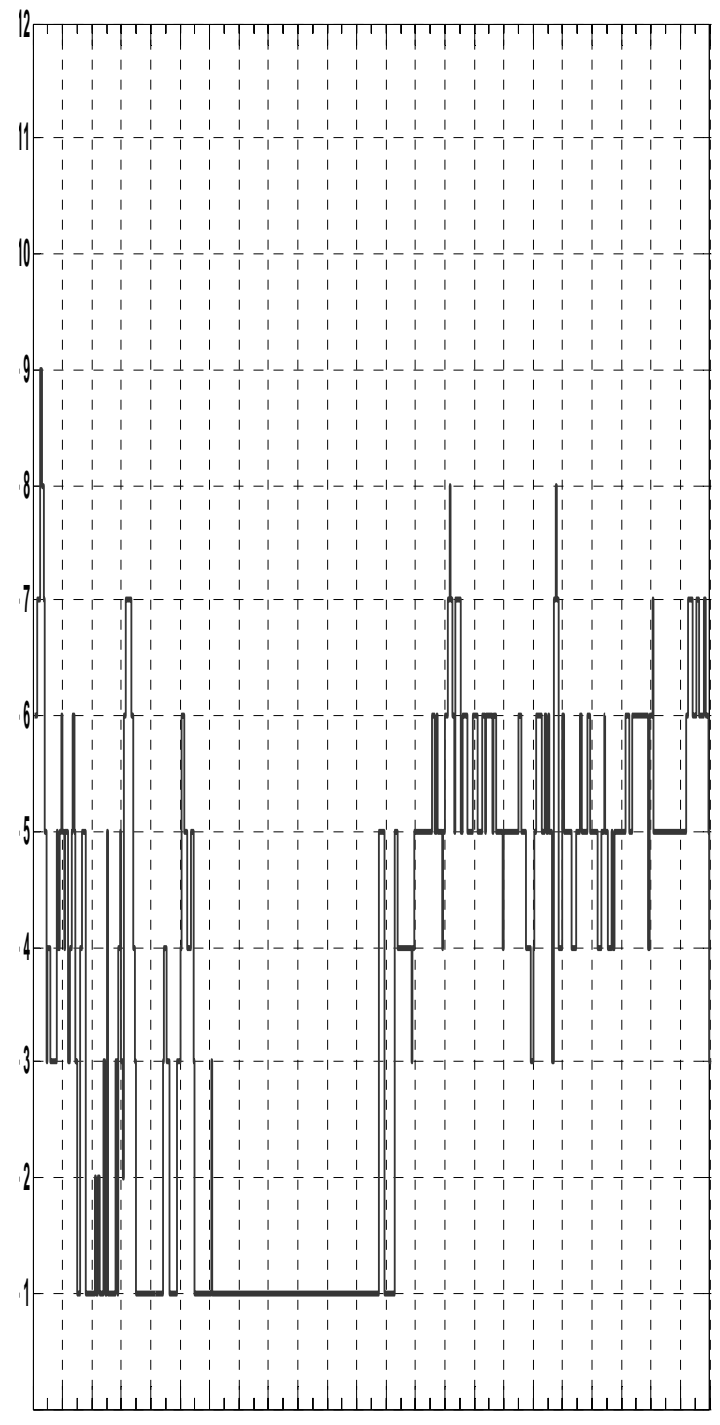

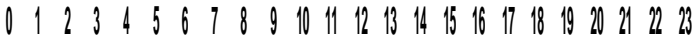

Figura 7 - Classificação do solo (tipo 1 a 12) até a profundidade de $23 \mathrm{~m}$

\section{6 - BIBLIOGRAFIA}

ABNT - ASSOCIAÇÃO BRASILEIRA DE NORMAS TÉCNICAS. SOLO Ensaio de penetração de cone in situ CPT. Método de ensaio. Rio de Janeiro: NBR 12069/91, 1991, 10 p.

BARNES G. Soil Mechanics: Principles and Practice, Palgrave Macmillan, 2000.

BEGEMANN, H. K. S. Improved method of determining resistance to adhesion by sounding through a loose sleeve placed behind the cone In Proceedings of the 3rd International Conference on Soil Mechanics and Foundation Engineering, ICSMFE, August 16 - 27, Zurich, Vol. 1, 1953, pp. $213-217$.

BEGEMANN, H. K. S. The use of the static penetrometer in Holland In New Zealand Engineering, Vol. 18, No. 2, 1963, p. 41.

BEGEMANN, H. K. S. The friction jacket cone as an aid in determining the soil profile In Proceedings of the 6th International Conference on Soil Mechanics and Foundation Engineering, ICSMFE, Montreal, September 8 - 15, Vol. 2, 1965, pp. 17 - 20.

BUDHU M. Soil Mechanics and Foundations, John Wiley \& Sons Inc. 2006.

CAMPANELLA, R G., GILLESPIE, D., AND ROBERTSON, P. K. Pore pressures during cone penetration testing In Proceedings of the 2nd European Symposium on Penetration Testing, ESOPT-2, Amsterdam, May 24 - 27, Vol. 2, 1982, pp. 507 - 512.

CAMPANELLA, R. G., AND ROBERTSON, P. K. Current status of the piezocone test In Proceedings of First International Symposium on Penetration Testing, ISOPT-1, Orlando, March 22 - 24, Vol. 1, 1988, pp. 93 - 116.

CAMPANELLA, R. G., ROBERTSON, P. K., DAVIES, M. P., AND SY, A. Use of in-situ tests in pile design In Proceedings 
12th International Conference on Soil Mechanics and Foundation Engineering, ICSMFE, Rio de Janeiro, Brazil, Vol. 1, 1989, pp. 199 - 203.

CAMPANELLA, R. G. AND ROBERTSON, P. K. Current Status of the Piezocone Test - State of the Art Report, In Proceedings Of the I Int. Symposium on Penetration Testing, Orlando, vol.1, 1988 pp. 93-116.

CHEN WAI-FAH The Civil Engineering Handbook, CRC Press Inc.,U.S., 2002.

DANZIGER, B. R.; VELLOSO, D. DE A. Correlações entre SPT e os resultados de ensaios de penetração contínua In Congresso Brasileiro de Mecânica de Solos e Engenharia de Fundações, 8, 1986, Porto Alegre. Anais, Porto Alegre, 1986, p.103113.

DANZIGER, F. A. B. ET AL. CPT-SPT Correlations for some Brazilian residual soils In Robertson, P. K.; Mayne (Ed.). Geotechnical site Characterization. Rotterdam: Balkema, 1998, p.907-912.

DANZIGER, F. A. B. Desenvolvimento do Equipamento para Realização de Ensaio de Piezocone: Aplicação a argilas moles, 1990, Tese D. Sc. COPPE/UFRJ, 1990.

DAVID MUIR WOOD Geotechnical Modelling, Spon Press, 2004.

DAVIES, M. P. \& CAMPANELLA, R. G. Piezocone technology: downhole geophysics for the geoenvironmental characterization of soil In Symposium on The Application of Geophysics To Engineering and Environmental Problems, Sageep'95, 1995, Orlando. Proceedings... Orlando (Florida, USA), 1995, 11 p.

DOUGLAS, B. J., AND OLSEN, R. S. Soil classification using electric cone penetrometer. American Society of Civil Engineers, ASCE In Proceedings of Conference on Cone Penetration Testing and Experience, St. Louis, October 26 - 30, 1981, pp. 209 - 227.
DAYHOFF, J. E. Neural Network Architectures an introduction. Van Nostrand Reinhold, 1990.

ESLAMI, A., AND FELLENIUS, B. H. Toe bearing capacity of piles from cone penetration test (CPT) data In Proceedings of the International Symposium on Cone Penetration Testing, CPT 95, Linköping,Sweden, October 4 - 5, Swedish Geotechnical Institute, SGI, Report 3:95, Vol. 2, 1995, pp. 453 - 460.

ESLAMI, A., AND FELLENIUS, B. H. Pile shaft capacity determined by piezocone (CPTu) data In Proceedings of 49th Canadian Geotechnical Conference, September 23 - 25, St. John's, Newfoundland, Vol. 2, 1996, pp. 859 867.

ESLAMI, A. Bearing capacity of piles from cone penetrometer test data, 1996, $516 \mathrm{p}, \mathrm{Ph}$. D. Thesis University of Ottawa, Department of Civil Engineering, 1996.

ESLAMI, A., AND FELLENIUS, B. H. Pile capacity by direct CPT and CPTu methods applied to 102 case histories In Canadian Geotechnical Journal, Vol. 34, No. 6, 1997, pp. 880 - 898.

FUGRO ENGINEERS B.V. Piezo-cone penetrometer - ISSUE - 3, 2000. Disponível em http://www.fugroengineers.nl/.

GREGG IN SITU INC. Cone Penetration Test Data Interpretation, 1998. Disponível em http: //www.greggdrilling.com/.

HAYKIN S. Neural networks a comprehensive foundation. Macmillan College P. C., 1994

HEAD K. H. Manual of Soil Laboratory Testing: Soil Classification and Compaction Tests Pt. 1, Whittles Publishing, 2006.

KAMESWARA RAO N. S. V. Dynamic Soil Tests and Applications, A H Wheeler Publishing Co Ltd, 2002.

HSAI-YANG FANG, JOHN L. DANIELS Introductory Geotechnical Engineering: An 
Environmental Perspective, Taylor \& Francis, 2006.

PRAKASH S., SHARMA H. D. Pile Foundations in Engineering Practice, John Wiley \& Sons Inc, 1990.

QUARESMA, A. R.; DECOURT, L.; QUARESMA FILHO, A. R.; ALMEIDA, M. S. S.; DANZIGER, F. Investigações geotécnicas In Hachich, W.; Falconi, F.; Saes. J.; Frota, R.; Carvalho, C.; Sussumu (Coordenadores), Fundações: teoria e prática. São Paulo, Editora PINI, Cap. 3, 1996, p. 119-162.

RIEDMILLER, M., AND H. BRAUN, A direct adaptive method for faster backpropagation learning: The RPROP algorithm In Proceedings of the IEEE International Conference on Neural Networks, San Francisco, 1993, pp. 234241.

ROBERTSON, P. K. AND CAMPANELLA, R. G. Interpretation of cone penetrometer tests in Part I sand. Canadian Geotechnical Journal, Vol. 20, No. 4, 1983, pp. 718 - 733.

ROBERTSON, P. K. AND CAMPANELLA, R. G. Guidelines for use, interpretation, and application of the CPT and CPTU In Manual, Hogentogler \& Company, Inc., 1986, 196 p.

ROBERTSON, P. K., CAMPANELLA, R. G., GILLESPIE, D., AND GRIEG, J. Use of piezometer cone data In Proceedings of American Society of Civil Engineers, ASCE, In-Situ 86 Specialty Conference, Edited by S. Clemence, Blacksburg, June 23 - 25, Geotechnical Special Publication GSP No. 6, 1986, pp. 1263 - 1280.

ROBERTSON, P. K. Soil classification using the cone penetration test In Canadian Geotechnical Journal, Vol. 27, No. 1, 1990, pp. $151-158$.

ROCHA FILHO, P. Behaviour in Cone Penetration Tests in Saturated Sands, 1982, PhD Thesis, University of London, 1982.
RUITER DE, J. Eletric Penetrometer for Site Investigation In Journal of the Soil Mechanics and Foundation Engineering, ASCE, SM2, 1971, pp. 457-473.

SANGLERAT, G., NHIM, T. V., SEJOURNE, M., AND ANDINA, R. Direct soil classification by static penetrometer with special friction sleeve In Proceedings of the First European Symposium on Penetration Testing, ESOPT-1, June 5 - 7, Stockholm, Vol. 2.2 , 1974, pp. 337 - 344.

SCHMERTMANN, J. H. Guidelines for cone test, performance, and design. Federal Highway Administration In Report FHWA-TS-78209, Washington, 1978, $145 p$.

SCHOFIELD A. Disturbed Soil Properties and Geotechnical Design, Thomas Telford Ltd, 2005.

SEARLE, I. W. The Interpretation of Begemann Friction Jacket Cone Results to Give Soil Types and Design Parameteres. Proceedings of the Symposuim on Strength Testing of Marine Sediments: Laboratory and In-Siotu Measurements. ASTM 04883000-38, San Diego, 1979, pp. 31-54.

SOARES, M. M., LUNNE, T., ALMEIDA, M. S. S., DANZIGER, F. A. B. Ensaios com Piezocones COPPE e Fugro em Argila Mole In Anais, VI COBRAMSEF, Porto Alegre, Vol. II, 1986, pp. 75-87.

TERZAGHI, K., 1930, Die Tragfahigkeit von Pfahlgrundungen In Die Bautechnik, citado por Velloso (1959).

VELloso, D. A., $O$ Ensaio de Diepsondering e a Determinação da Capacidade de Carga do Solo In Rodovia, No. 29, Rio de Janeiro, 1959, pp. 3-7.

VOS, J. D. The practical use of CPT in soil profiling In Proceedings of the Second European Symposium on Penetration Testing, ESOPT-2, Amsterdam, May 24 27, Vol. 2, 1982, pp. 933 - 939.

WALTHAM T. Foundations of Engineering Geology, Spon Press, 2001. 
WOODWARD J. Introduction to Geotechnical Processes, Taylor \& Francis, 2004.

ZURADA, J. M. Introduction to artificial neural systems. West Publishing Company, 1992. 